\title{
Caracterización física y química de ríos de montaña (Tafí del Valle-Tucumán-Argentina)
}

\author{
Sara C. Isasmendi ${ }^{1,2, *}$, Beatriz C. Tracanna ${ }^{1,2,3}$, Francisco H. Vendramini ${ }^{4}$, María G. Navarro ${ }^{5,6}$, \\ María A. Barrionuevo ${ }^{5}$ y Gladys S. Meoni ${ }^{5}$
}

1 Instituto de Limnología del Noroeste Argentino (ILINOA), Facultad de Ciencias Naturales e Instituto Miguel Lillo, Universidad Nacional de Tucumán. Miguel Lillo 205 (4000) San M. de Tucumán, Argentina.

2 Fundación Miguel Lillo. Miguel Lillo 251 (4000) San M. de Tucumán, Argentina. tracanna@csnat.unt.edu.ar

3 Consejo Nacional de Investigaciones Científicas y Técnicas (CONICET)

${ }^{4}$ Laboratorio de Química Analítica. Facultad de Bioquímica, Química y Farmacia, Universidad Nacional de Tucumán. Ayacucho 451 (4000) San M. de Tucumán, Argentina. fvendramini@ fbqf.unt.edu.ar

5 Laboratorio de Control Ambiental, SIPROSA. Pje. Dorrego 1080 (4000) San M. de Tucumán, Argentina. smeoni@hotmail.com

${ }^{6}$ Facultad de Agronomía y Zootecnia, Universidad Nacional de Tucumán. Av. Roca 1900 (4000) San M. de Tucumán, Argentina.gmansilla@ herrera.unt.edu.ar

* Autor responsable de la correspondencia: sarisa@ csnat.unt.edu.ar

\section{RESUMEN}

\section{Caracterización física y química de ríos de montaña (Tafí del Valle-Tucumán-Argentina)}

En este trabajo se estudió durante el ciclo anual 2000-2001, a los ríos Blanquito, Churqui, de La Ovejería, de Las Carreras, Tafí, El Mollar y Los Sosa, ubicados a 2000 msnm en el valle de Tafí (Tucumán-Argentina). El objetivo de esta contribución fue caracterizar e interpretar las variaciones espacio-temporales de los parámetros físicos y químicos en relación con la dinámica hidrológica a la que se encuentran sujetos estos ecosistemas acuáticos y además, comparar la calidad de sus aguas. Las variables físicas y químicas analizadas fueron: oxígeno disuelto $(\mathrm{OD})$, demanda bioquímica de oxígeno $\left(\mathrm{DBO}_{5}\right)$, iones mayoritarios, compuestos nitrogenados, ortofosfatos y metales pesados. Los resultados muestran que la temperatura varió entre 4.2 y $28{ }^{\circ} \mathrm{C}$, el pH fluctuó de neutro a alcalino (6.7-8.7) y la conductividad eléctrica entre 64 y $296 \mu \mathrm{S} / \mathrm{cm}$. El OD y la $\mathrm{DBO}_{5}$ se mostraron estables, alcanzando máximos valores de 10.1 y $2 \mathrm{mg} / \mathrm{l}$, respectivamente. El agua pudo caracterizarse como bicarbonatada-cálcica dominante. Las concentraciones de $\mathrm{N}_{-} \mathrm{NO}_{3}^{-}$oscilaron entre 0.1 y $0.6 \mathrm{mg} / \mathrm{l}$, el N-NO $\mathrm{NO}_{2}^{-}$sólo llegó en el río Churqui a $0.01 \mathrm{mg} / \mathrm{l}$ y el N-NH+ alcanzó valores de $0.8 \mathrm{mg} / \mathrm{l}$. En general los registros de los ortofosfatos fueron inferiores a $0.2 \mathrm{mg} / \mathrm{l}$. Los metales pesados $\mathrm{Cu}^{2+}$ y Fe${ }^{3+}$ llegaron a un máximo de $3 \mathrm{mg} / \mathrm{l}$ y $1.8 \mathrm{mg} / \mathrm{l}$, respectivamente. Las mayores concentraciones del hierro se presentaron en el río de La Ovejería y de cobre en el río El Mollar, probablemente por contaminación antropogénica. La interpretación de los análisis de componentes principales realizados sobre la matriz de parámetros físicos y químicos, permitió diferenciar a los sistemas lóticos temporal y espacialmente. El componente 1 ordenó a los ríos de acuerdo a los iones mayoritarios predominantes y a la conductividad, lo que podría interpretarse como un eje de mineralización. Los componentes 2 y 3 , ordenaron las muestras de invierno y primavera y las de verano y otoño, de acuerdo a la variación cíclica estacional coincidiendo con las condiciones climáticas del área de estudio.

Palabras clave: Hidroquímica, sistemas lóticos, calidad del agua, Noroeste Argentino.

\section{ABSTRACT}

\section{Physical and chemical characterization of mountain rivers (Tafi del Valle-Tucumán-Argentina)}

In this work, throughout the 2000-2001 annual period, the Blanquito, Churqui, de La Ovejería, de Las Carreras, Tafi, El Mollar and Los Sosa rivers, located at $2000 \mathrm{~m}$ above sea level in the Tafi Valley (Tucumán-Argentina) were studied. The aim of this paper was not only to characterize and understand the spatial and temporal variations of physical and chemical parameters related to the hydrological dynamics these aquatic ecosystems are subject to, but also to compare the quality of their waters. Physical and chemical variables analysed were: dissolved oxygen $(D O)$, biochemical oxygen demand $\left(B O D_{5}\right)$, preponderant ions, nitrogenous compounds, orthophosphates, and heavy metals. Results show that the temperature ranged 
between 4.2 and $28^{\circ} \mathrm{C}$, pH fluctuated from neutral to alkaline (6.7-8.7) and electrical conductivity varied between 64 and $296 \mu \mathrm{S} / \mathrm{cm}$. DO and $\mathrm{BOD}_{5}$ were stable and reached maximum values of 10.1 and $2 \mathrm{mg} /$, respectively. The water could be characterized as mainly bicarbonated-calcic. $\mathrm{N}-\mathrm{NO}_{3}^{-}$concentrations ranged between 0.1 and $0.6 \mathrm{mg} / \mathrm{l}, \mathrm{N}-\mathrm{NO} \mathrm{O}_{2}^{-}$reaching only $0.01 \mathrm{mg} / \mathrm{l}$ in the Churqui river, and $\mathrm{N}_{-} \mathrm{NH}_{4}^{+}$reached values of $0.8 \mathrm{mg} /$. In general, orthophosphate records were lower than $0.2 \mathrm{mg} / \mathrm{l}$. Maximum values for heavy metals, $\mathrm{Cu}^{2+}$ and $\mathrm{Fe}^{3+}$ were $3 \mathrm{mg} / \mathrm{l}$ y $1.8 \mathrm{mg} /$, respectively. Iron highest concentrations were found in de La Ovejería river, whereas those for copper were found in El Mollar river, probably due to anthropogenic pollution. The interpretation of the main component analyses carried out on the physical and chemical parameters matrix, allowed to differentiate lotic systems both temporally and spatially. Component 1 ordered the rivers according to both the prevailing ions and conductivity, which could be interpreted as a mineralization axis. Components 2 and 3 ordered the winter and spring, and the summer and autumn samples according to the seasonal cyclic variation, coinciding with the climatic conditions of the area under study.

Keywords: Hydrochemistry, lotic systems, water quality, northwestern Argentina.

\section{INTRODUCCIÓN}

Argentina es rica en ambientes acuáticos, pero no todas las regiones poseen una adecuada distribución del agua. En la mitad norte los humedales son más abundantes en el este y en la mitad sur en el oeste, sin considerar los costeros. Los humedales de las zonas áridas y semiáridas tienen un valor especial pues allí el agua suele ser un limitante para el desarrollo de la vida y de las actividades humanas (Canevari et al., 1998). En el país se diferencian seis regiones que agrupan humedales con características ecológicas similares. El sistema Salí-Dulce, incluido en los humedales de la región del Chaco, desemboca en la laguna de Mar Chiquita y forma parte de la mayor cuenca cerrada de América del Sur (Bucher y Chani, 1998). Existen numerosos antecedentes químicos y biológicos relacionados a los ríos de la parte superior de la Cuenca Salí-Dulce, destacándose los de Fernández et al. (2002), Mirande et al. (1999), Perondi et al. (1996), Seeligmann (1999), Seeligmann et al. (2001), Tracanna y Martinez De Marco (1997), Tracanna et al. (1999) y Villagra de Gamundi et al. (1993), entre otros.

El área de estudio, valle de Tafí, se encuentra ubicado en Tucumán, noroeste argentino. En los ambientes acuáticos de esta zona (parte superior de la Cuenca Salí-Dulce), sólo existen datos físicos, químicos y biológicos del embalse La Angostura (Acosta et al., 2002; Locascio de Mitrovich et al.,
1997; Seeligmann et al., 2003, 2004; Tracanna y Seeligmann, 2004), no habiendo antecedentes referidos a los ríos del valle. Asimismo, Locascio de Mitrovich et al. (1997) estimaron deterioro del agua a corto plazo, ya que la atracción turística que ofrece la zona, ha provocado el crecimiento de las poblaciones próximas a dicho embalse, surgiendo graves problemas como la falta de tratamiento de los residuos domésticos, a los que se agregan los generados por la extensión de la actividad agrícola y la aplicación de pesticidas y fertilizantes.

Este trabajo forma parte de un proyecto mas amplio que incluyen aspectos biológicos de los ambientes lóticos analizados. Los objetivos específicos son caracterizar a siete ríos de montaña a fin de interpretar las variaciones espacio-temporales de los parámetros físicos y químicos en relación con la dinámica hidrológica a la que se encuentran sujetos estos ecosistemas acuáticos y además, comparar la calidad de sus aguas.

\section{ÁREA DE ESTUDIO}

El valle de Tafí, con una área de $700 \mathrm{~km}^{2}$ (García Salemi, 1974), se encuentra ubicado al oeste de la provincia de Tucumán (Fig. 1A), en la unidad hidrográfica Cuenca media del río Salí, Subcuenca Balderrama (Alderete, 1998).

Los límites del valle son las Cumbres de Tafí en el este, al oeste el cerro Muñoz, al norte las Cumbres Calchaquíes y al sur el cerro Nuñorco 


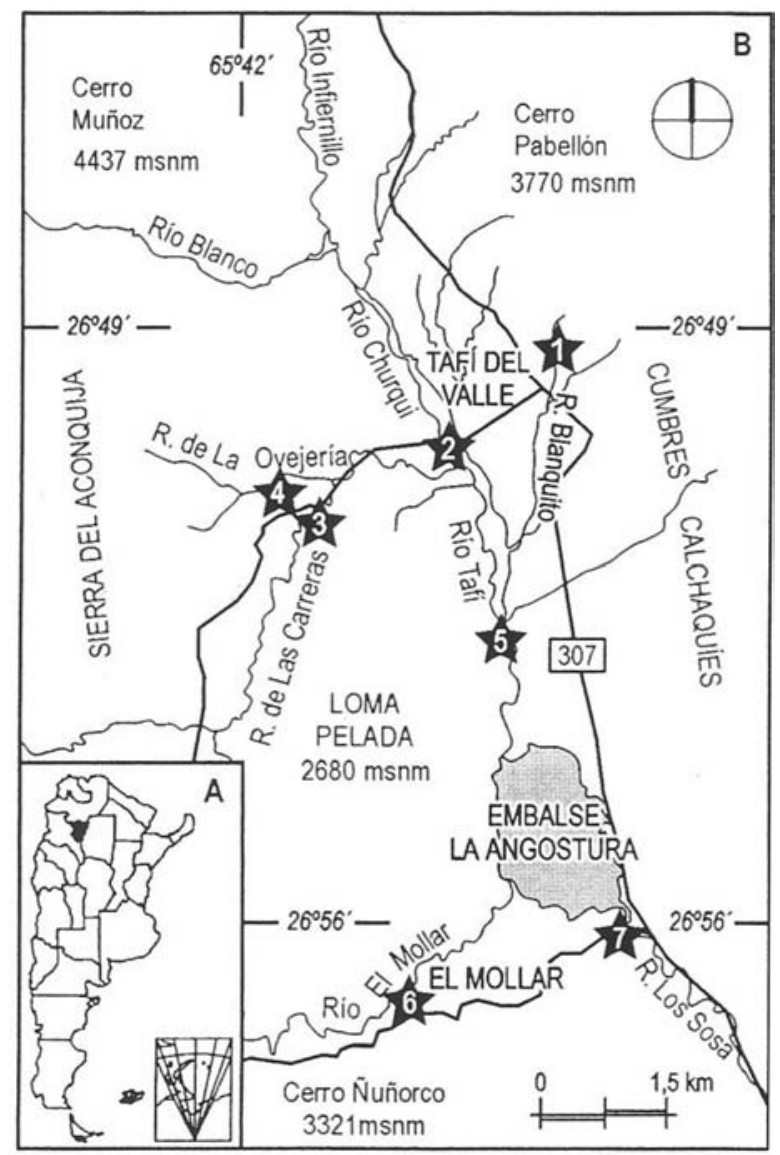

Figura 1. A-Ubicación de Tucumán, Argentina. B-Área de estudio y sitios de muestreo: 1-R. Blanquito; 2-R. Churqui; 3-R. de Las Carreras; 4-R. de La Ovejería; 5-R. Tafí; 6-R. El Mollar y 7-R. Los Sosa. A-Location of Tucumán, Argentina. B-Study area and sampling sites: 1-R. Blanquito; 2-R. Churqui; 3-R. de Las Carreras; 4-R. de La Ovejería; 5-R. Tafí; 6-R. El Mollar and 7- R. Los Sosa.

Grande (perteneciente a la Sierra del Aconquija). En su parte central se ubica la Loma Pelada, con una altura máxima de $2680 \mathrm{msnm}$. El principal colector del valle es el río Tafí, que recibe de su margen izquierdo algunos cursos de agua que descienden de las Cumbres Calchaquíes, como al Arroyo La Quebradita (conocido como río Blanquito), el cual abastece a una de las plantas potabilizadoras de la población de Tafí del Valle. Por su margen derecha ingresan, entre otros, los ríos de La Ovejería y de Las Carreras. El río Tafí, al igual que el río El Mollar que desciende del sudoeste, desembocan en el Embalse La
Angostura. Éste es de forma subtriangular, tiene una superficie de $800 \mathrm{ha}$, un volumen de $1600 \mathrm{hm}^{3}$, su profundidad y longitud máximas son de $32 \mathrm{~m}$ y $4800 \mathrm{~m}$, respectivamente (Locascio de Mitrovich et al., 1997). Aguas debajo del embalse, el río recibe el nombre de Los Sosa (Fig. 1B).

El valle de Tafí está limitado por bloques de basamento metamórfico del Paleozoico inferior, afectados por intrusiones graníticas del Paleozoico medio, correspondientes a la Sierra del Aconquija y a las Cumbres Calchaquíes. Por encima de este basamento, se encuentran relictos de una cubierta sedimentaria de edad terciaria que se observa al este de La Angostura. El relleno cuaternario del valle esta representado por sedimentos aluvionales gruesos, los cuales constituyen conos (antiguos y actuales) y glacís antiguos por depósitos de loess (Tineo et al., 1998).

El loess es sometido durante todo el año a la influencia eólica que produce un retransporte del material, intensificándose en el área de La Angostura durante la estación seca. El valle se encuentra bajo un severo riesgo de erosión laminar, acentuado por el clima semiárido y la presencia de suelos loésicos, sumado al sobrepastoreo y al cultivo irrestricto (Sayago et al., 1998).

El material de granulometría gruesa está formado principalmente por clastos de origen sedimentario, los que fueron polideformados y metamorfizados en sucesivos eventos geológicos, mientras que el de granulometría fina de naturaleza loésica contiene más de $50 \%$ de limo, constituido por minerales arenosos y arcillosos (Sayago et al., 1998).

El clima de la región presenta inviernos secos y veranos templados, con una temperatura media anual de $18{ }^{\circ} \mathrm{C}$ y precipitaciones que llegan a 400 $\mathrm{mm}$ anuales, produciéndose las máximas en el mes de enero, variando entre 80 y $100 \mathrm{~mm}$.

El área de estudio pertenece fitogeográficamente a la provincia de las Yungas, Distrito de los Bosques Montanos (Cabrera, 1976) y según Vervoorst (1981) a la comunidad vegetal denominada pastizal de altura y matorrales mesofíticos. El pastizal natural se ha modificado por la actividad antrópica: principalmente por prácticas de quema, actividad agrícola y ganadera. 


\section{MATERIAL Y MÉTODOS}

Se efectuaron trece campañas mensuales entre octubre de 2000 y 2001 en las siguientes estaciones de muestreo: 1.- río Blanquito $\left(26^{\circ} 49^{\prime}\right.$ $\left.\mathrm{S}, 65^{\circ} 42^{\prime} \mathrm{W}\right), 2350 \mathrm{msnm}$; 2.- río Churqui $\left(26^{\circ} 50^{\prime} \mathrm{S}, 65^{\circ} 43^{\prime} \mathrm{W}\right), 2150 \mathrm{msnm}$; 3.- río de Las Carreras $\left(26^{\circ} 51^{\prime} \mathrm{S}, 6^{\circ} 44^{\prime} \mathrm{W}\right), 2170 \mathrm{msnm}$; 4.río de La Ovejería $\left(26^{\circ} 51^{\prime} \mathrm{S}, 6^{\circ} 44^{\prime} \mathrm{W}\right), 2200$ msnm; 5.- río Tafí $\left(26^{\circ} 53^{\prime} \mathrm{S}, 65^{\circ} 42^{\prime} \mathrm{W}\right), 1950$ msnm; 6.- río El Mollar $\left(26^{\circ} 56^{\prime} \mathrm{S}, 65^{\circ} 42^{\prime} \mathrm{W}\right)$, 2050 msnm y 7.- río Los Sosa $\left(26^{\circ} 56^{\prime} \mathrm{S}, 65^{\circ} 40^{\prime}\right.$ W), 1990 msnm (Fig. 1B).

Los parámetros físicos y químicos: temperatura del agua, $\mathrm{pH}$, conductividad, velocidad de la corriente y profundidad se determinaron "in situ". Se midieron temperatura y $\mathrm{pH}$ utilizando un peachímetro digital portátil Altronix, modelo TPA-1 y conductividad eléctrica con un conductímetro Altronix, modelo CT-1. La medición de la velocidad de la corrien- te se realizó por el método de flotación y la profundidad de los ríos fue tomada en la zona media. La posición de los sitios de muestreo se localizó mediante un GPS 12 XL.

Las muestras para el análisis de oxígeno disuelto (OD) se fijaron en el campo y se determinaron en laboratorio, mientras que para los análisis químicos de iones mayoritarios, demanda bioquímica de oxígeno $\left(\mathrm{DBO}_{5}\right)$, compuestos nitrogenados, ortofosfatos y metales pesados se extrajeron en recipientes plásticos de 1.5 litros, lavados previamente con agua destilada. Para el análisis de estos últimos se utilizó ácido nítrico diluido en una proporción 3:1 y se fijaron en campo con 5 gotas de ácido nítrico concentrado. Todas las muestras se trasladaron al laboratorio en frío y oscuridad.

Los iones mayoritarios se midieron cinco veces durante el ciclo hidrológico (octubre/00 $\mathrm{y}$ enero, abril, julio, octubre/01), mientras que los demás parámetros se determinaron en forma

Tabla 1. Variables físicas, químicas y métodos utilizados. Physical and chemical variables, and methods used.

\begin{tabular}{|c|c|c|}
\hline VARIABLES & UNIDADES & MÉTODOS ANALÍTICOS \\
\hline Temperatura & ${ }^{\circ} \mathrm{C}$ & Termómetro \\
\hline Conductividad & $\mu \mathrm{S} / \mathrm{cm}$ & Conductimétrico \\
\hline $\mathrm{pH}$ & & Potenciométrico \\
\hline Profundidad & $\mathrm{cm}$ & Ruleta \\
\hline Velocidad de la corriente & $\mathrm{m} / \mathrm{seg}$ & Método de flotante \\
\hline Alcalinidad & $\mathrm{mg} / \mathrm{l}$ & Volumétrico ácido-base \\
\hline Sodio y potasio & $\mathrm{mg} / \mathrm{l}$ & Fotometría de emisión \\
\hline Calcio & $\mathrm{mg} / \mathrm{l}$ & Complexométrico con reactivo EDTA y murexide en polvo \\
\hline Magnesio & $\mathrm{mg} / \mathrm{l}$ & Complexométrico con reactivo EDTA y negro de eriocromo $\mathrm{T}$. \\
\hline Cloruro & $\mathrm{mg} / \mathrm{l}$ & Volumétrico (Mohr) \\
\hline Sulfato & $\mathrm{mg} / \mathrm{l}$ & Nefelométrico \\
\hline Nitrógeno de amonio & $\mathrm{mg} / \mathrm{l}$ & Espectrofotométria visible con reactivo de Nessler \\
\hline Nitrógeno de nitrato & $\mathrm{mg} / \mathrm{l}$ & Espectrofotométria visible con ácido Fenoldisulfonico \\
\hline Nitrógeno de nitrito & $\mathrm{mg} / \mathrm{l}$ & Espectrofotometría visible con Clorhidrato de naftilamina \\
\hline Ortofosfato & $\mathrm{mg} / \mathrm{l}$ & Espectrofotométria de reducción con cloruro estagnoso \\
\hline Oxígeno disuelto & $\mathrm{mg} / \mathrm{l}$ & Determinación de Iodo con Tiosulfato. Winkler \\
\hline Demanda bioquímica de oxígeno & $\mathrm{mg} / \mathrm{l}$ & Determinación de Iodo con Tiosulfato. Método de dilución \\
\hline Arsénico & $\mathrm{mg} / \mathrm{l}$ & Espectrofotometría visible con dietil-ditiocarbamato de plata y efedrina \\
\hline Cadmio & $\mathrm{mg} / \mathrm{l}$ & Espectrofotometría visible con ditisona \\
\hline Cinc & $\mathrm{mg} / \mathrm{l}$ & Espectrofotometría visible con ditisona \\
\hline Cobre & $\mathrm{mg} / \mathrm{l}$ & Espectrofotometría visible con neocuproína \\
\hline Hierro & $\mathrm{mg} / \mathrm{l}$ & Espectrofotometría visible con fenantrolina \\
\hline Manganeso & $\mathrm{mg} / \mathrm{l}$ & Espectrofotometría visible con persulfato \\
\hline
\end{tabular}


mensual. Para octubre de 2001 no se presentaron datos en el río El Mollar debido a que su caudal fue desviado para riego.

Para la elaboración de los gráficos radiales, se calcularon los valores promedios del número de miliEquivalente de los iones mayoritarios presentes $\left(\mathrm{HCO}_{3}^{-}, \mathrm{SO}_{4}^{2-}, \mathrm{Cl}^{-}, \mathrm{Ca}^{2+}, \mathrm{Mg}^{2+}, \mathrm{Na}^{+}\right.$y K $\left.{ }^{+}\right)$.
La metodología que se siguió para los análisis físicos y químicos fue la recomendada en APHA (1992) y se muestran en la Tabla 1.

$\mathrm{El}$ análisis de componentes principales (ACP) se efectuó con los siguientes parámetros: temperatura (Tem.), profundidad (Prof.), $\mathrm{pH}$, conductividad (Cond.), iones mayoritarios, $\mathrm{OD}, \mathrm{DBO}_{5}$,
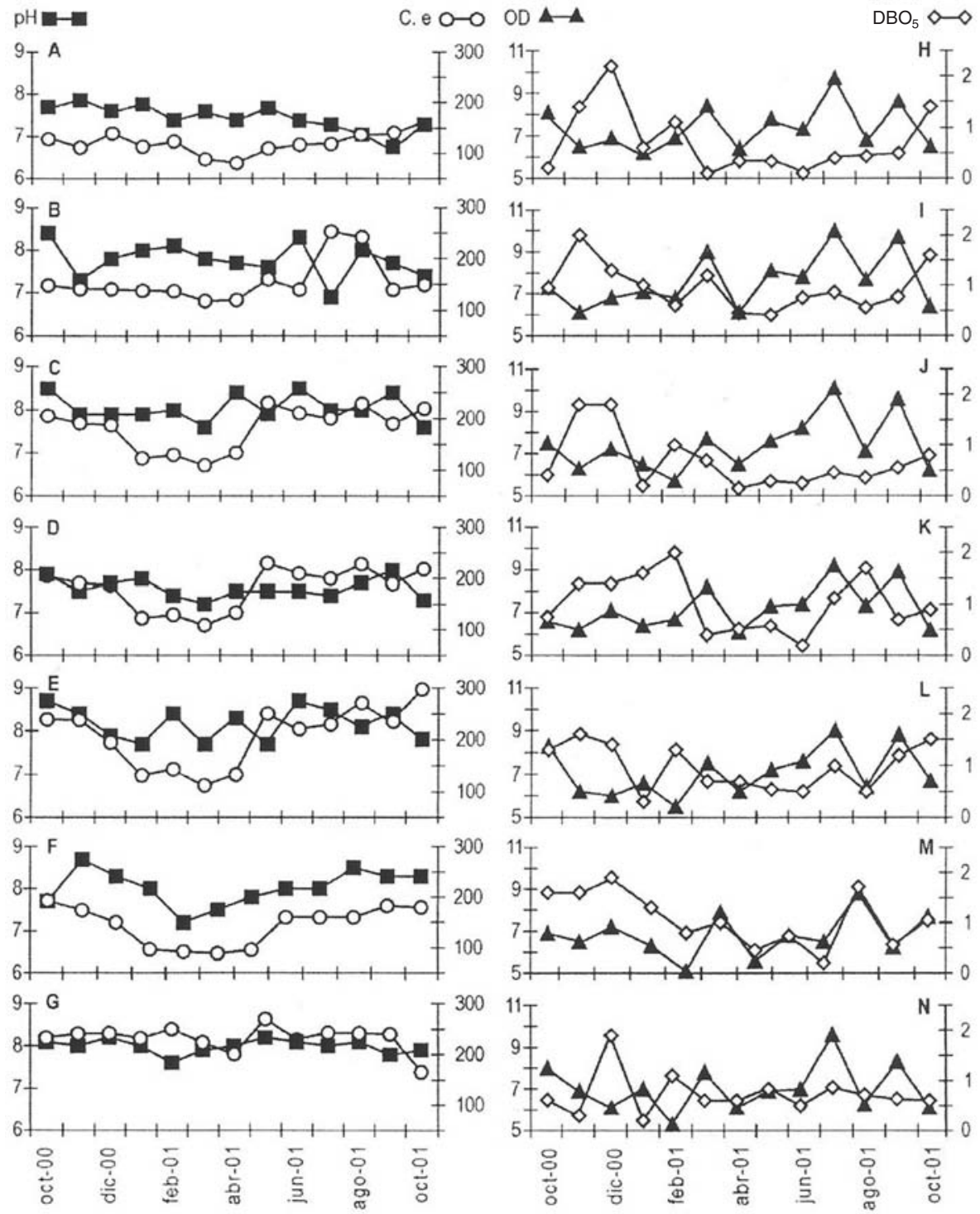

Figura 2. Variación anual de: $\mathrm{pH}$ y conductividad $(\mu \mathrm{S} / \mathrm{cm})(\mathrm{A}-\mathrm{G})$; oxígeno disuelto y demanda bioquímica de oxígeno (mg/l) (HN). A y H: R. Blanquito; B e I: R. Churqui; C y J: R. de Las Carreras; D y K: R. de La Ovejería; E y L: R. Tafí; F y M: R. El Mollar y $G$ y N: R. Los Sosa. Annual variation of: $p H$ and conductivity $(\mu S / \mathrm{cm})(A-G)$; dissolved oxygen and biochemical oxygen demand (mg/l) (H-N). A and H: R. Blanquito; B and I: R. Churqui; C and J: R. de Las Carreras; $D$ and K: R. de La Overjería; E and L: R. Tafi; F and M: R. El Mollar and G and N: R. Los Sosa. 


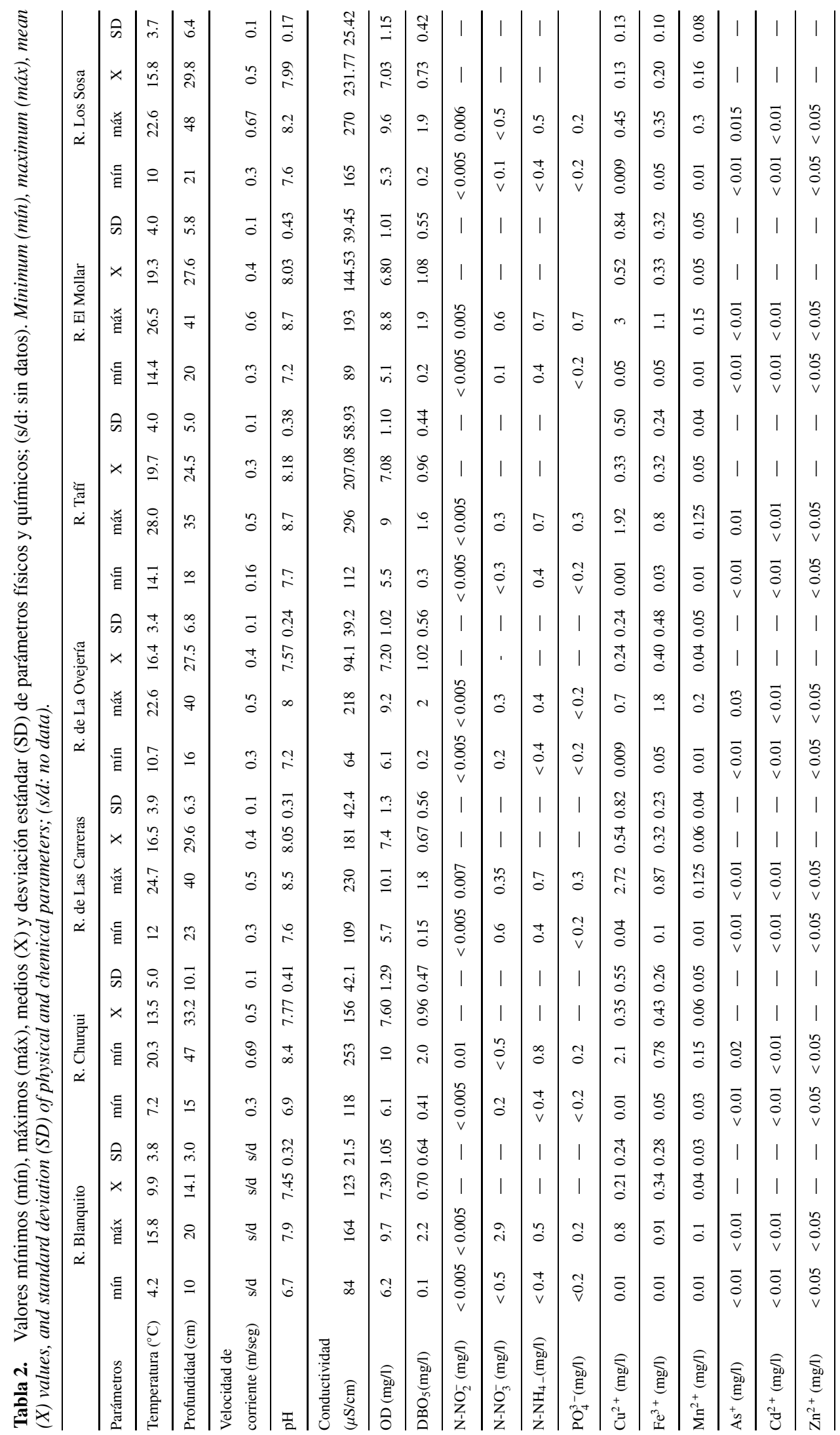


compuestos nitrogenados, ortofosfatos y metales pesados (valores normalizados y estandarizados) y se trabajó con la matriz de correlación. Los datos se procesaron mediante el paquete estadístico NTSYS (Rohlf, 1990).

Asimismo, se efectuó un análisis de correlación (coeficiente de Pearson), haciendo uso del programa SPSS, entre las variables físicas y químicas de todos los ríos y de cada uno de ellos en particular. Cabe destacar que los resultados más relevantes de éstos últimos, se detallan en el texto sin incluir las tablas respectivas. Previamente los datos fueron transformados logarítmicamente, con excepción del pH. En este trabajo se comentan aquellas correlaciones que resultaron significativas $(p<0.05 \mathrm{y}$ $p<0.01)$ de todos los ríos y los resultados relevantes de los análisis individuales.

\section{RESULTADOS}

Los registros mínimos, máximos, medios y desviación estándar de las variables analizadas de los ambientes lóticos estudiados, excepto los iones mayoritarios, se presentan en la Tabla 2. La temperatura del agua osciló entre $4.2{ }^{\circ} \mathrm{C}$ (río Blanquito, mayo/01) y $28{ }^{\circ} \mathrm{C}$ (río Tafí, enero-febrero/01), a menudo fue similar entre los puntos de muestreo, con excepción de los ríos Blanquito y Los Sosa, donde se presentaron los valores más bajos. La profundidad de los ríos fluctuó entre 10 y $48 \mathrm{~cm}$, encontrándose en el Blanquito los mínimos registros. En el río Tafí en octubre/01 se presentó el menor valor de velocidad de la corriente de $0.16 \mathrm{~m} / \mathrm{seg}$ y el máximo con $0.69 \mathrm{~m} / \mathrm{seg}$ en el río Churqui en mayo/01. La correlación entre profundidad y temperatura fue significativa $(r=0.36 ; p<0.05)$.

El pH varió de neutro a alcalino, desde 6.7 (setiembre/01) en el Blanquito a 8.7 (octubre/00 y junio/01) en el Tafí y en El Mollar (noviembre/00), siendo en el segundo río en general, superior a 8 (Fig. 2A-G). Asimismo, la correlación entre $\mathrm{pH}$ y temperatura fue altamente significativa $(r=0.52, p<0.01)$.

La conductividad (Fig. 2A-G) osciló entre 64$296 \mu \mathrm{S} / \mathrm{cm}$ en los ríos de La Ovejería y Tafí, respectivamente. En Los Sosa, con frecuencia, fue superior a 200 durante el período analizado. En los ríos Churqui, de Las Carreras, de La Ovejería, y Tafí se observó correlación negativa entre temperatura y conductividad cuyos valores fueron altamente significativa en el primero y significativa en los restantes respectivamente, $(r$ $=-0.51 ; r=-0.48 ; r=-0.46$ y $r=-0.65)$.

En el río Blanquito se registraron los máximos y mínimos valores de $\mathrm{DBO}_{5}$ con $2.2 \mathrm{mg} / \mathrm{l}$ en diciembre/00 y $0.1 \mathrm{mg} / \mathrm{l}$ en marzo y junio/01 (Fig. 2H). Los datos obtenidos de OD muestran un buen estado de oxigenación en todos los ríos (Fig. 2H-N) fluctuando entre 5.1 (río El Mollar, febrero/01) y $10.1 \mathrm{mg} / \mathrm{l}$ (río de Las Carreras, julio/01). La correlación entre OD con la temperatura y la conductividad fue altamente significativa en la mayoría de los ríos (Tabla 3), negativa con el primer parámetro excepto en Los Sosa y positiva con la segunda variable excluyendo a El Mollar y Los Sosa.

El análisis de la composición aniónica y catiónica para las cuatro estaciones del año, reflejan que las aguas de los ríos estudiados son bastante estables a lo largo del tiempo, con una composición bicarbonato-cálcica dominante (Fig. 3A-G). En los ríos de La Ovejería y Blanquito (Fig. 3D y A), se obtuvieron valores mínimos que oscilaron para $\mathrm{HCO}_{3}^{-}$entre $0.76 \mathrm{y}$ $1.04 \mathrm{mEq} / \mathrm{l}$ y para $\mathrm{Ca}^{2+}$ entre 0.4 y $0.65 \mathrm{mEq} / \mathrm{l}$. Los registros, en los restantes ríos, fueron para $\mathrm{HCO}_{3}^{-}$entre 1.6 y $2.11 \mathrm{mEq} / 1$ y para $\mathrm{Ca}^{2+}$ entre 0.93 y $1.02 \mathrm{mEq} / \mathrm{l}$ (Fig. 3B, C, E-G). En cuanto a los otros iones analizados, los valores obtenidos de cationes en forma creciente fueron: $\mathrm{K}^{+}, \mathrm{Mg}^{2+}, \mathrm{Na}^{+}$y de aniones $\mathrm{Cl}^{-}$y $\mathrm{SO}_{4}^{2-}$. La Tabla 3 muestra que la correlación, en general, fue altamente significativa entre los iones mayoritarios con excepción del sulfato. Los ríos Los Sosa y El Mollar no coincidieron con lo expresado anteriormente, en especial el primero no presentó correlación entre los cationes y aniones considerados. También en la misma tabla se observó que estos iones se correlacionaron en su mayoría con un $p<0.01$ con la conductividad y el pH. Ésto se cumplió especialmente con el primer parámetro en casi todos los ríos, exceptuando a Los Sosa. 
A

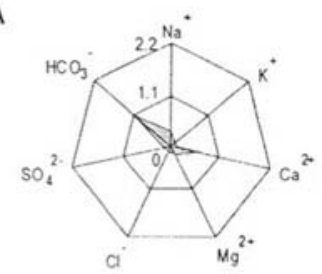

B

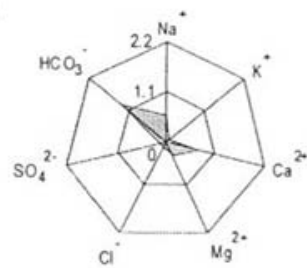

D

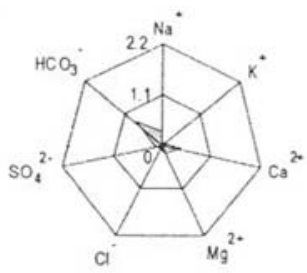

$\mathbf{F}$

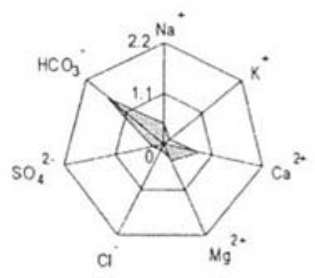

C

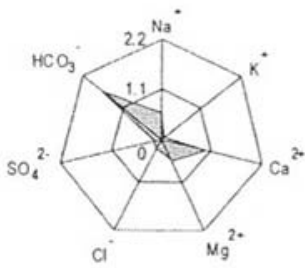

E
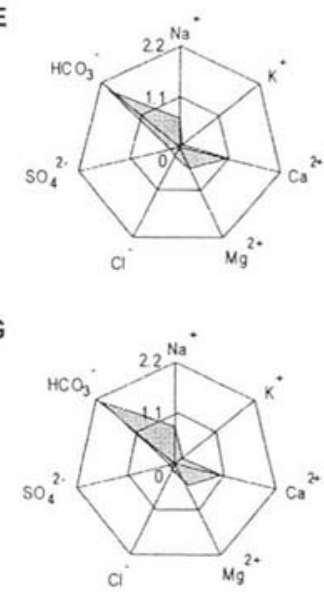

Figura 3. Balance promedio estacional de aniones y cationes (mEq/l) en: A-R. Blanquito; B-R. Churqui; C-R. de Las Carreras; D-R. de La Ovejería; E-R. Tafí; F-R. El Mollar y G-R. Los Sosa. Mean seasonal balance of anions and cations $(m E q /)$ in: A-R. Blanquito; B-R. Churqui; $C$-R. de Las Carreras; D-R. de La Ovejería; E-R. Tafí; F-R. El Mollar and G-R. Los Sosa.

Los registros de los componentes nitrogenados se mantuvieron constantes a lo largo del período estudiado. Las concentraciones de nitrógeno

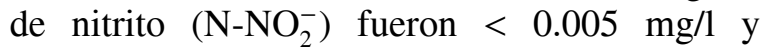
sólo alcanzaron en el río Churqui a $0.01 \mathrm{mg} / \mathrm{l}$ en diciembre/00, mientras las de nitrógeno de nitrato $\left(\mathrm{N}-\mathrm{NO}_{3}^{-}\right)$oscilaron frecuentemente, entre 0.1 y $0.6 \mathrm{mg} / \mathrm{l}$ y se encontró un máximo valor de $2.9 \mathrm{mg} / \mathrm{l}$ en agosto y setiembre/01 en el río Blanquito. El nitrógeno de amonio $\left(\mathrm{N}^{-} \mathrm{NH}_{4}^{+}\right)$llegó a valores de $0.8 \mathrm{mg} / \mathrm{l}$ en el río Churqui (diciembre/00) y a menudo los

ortofosfatos $\left(\mathrm{PO}_{4}^{3-}\right)$ se ubicaron por debajo de $0.2 \mathrm{mg} / \mathrm{l}$, con excepción de este último río donde alcanzó $0.7 \mathrm{mg} / \mathrm{l}$ en noviembre/00 (Tabla 2). Se observó correlación entre $\mathrm{N}^{-\mathrm{NO}_{3}^{-}}$/profundidad $(r$ $=-0.56 ; p<0.01)$, valor que correspondió solamente al río Blanquito (Tabla 3). En el análisis de cada río, se obtuvo en la mayoría de ellos, correlación significativa positiva entre $\mathrm{N}$ -

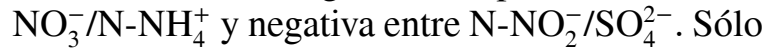
Los Sosa presentó correlaciones entre $\mathrm{N}^{-} \mathrm{NH}_{4}^{+} \mathrm{y}$ $\mathrm{PO}_{4}^{3-}(r=-0.99 ; p<0.01)$ y entre $\mathrm{N}-\mathrm{NO}_{3}^{-}$y $\mathrm{PO}_{4}^{3-}$ $(r=-0.89 ; p<0.05)$.

$\mathrm{El}$ arsénico registró máximos valores de 0.03 $\mathrm{mg} / \mathrm{l}$ en el río de La Ovejería en noviembre/00 y $0,02 \mathrm{mg} / \mathrm{l}$ en el río Churqui en diciembre/ 00 . El cobre llegó a un máximo de $3 \mathrm{mg} / \mathrm{l}$ en noviembre/00 en El Mollar y presentó tenores superiores a $2 \mathrm{mg} / \mathrm{l}$ en el de Las Carreras (octubre/00) y Churqui (noviembre/00) (Tabla 2, Fig. 4A-G). Los máximos registros de hierro se observaron en enero/01 en el río de $\mathrm{La}$ Ovejería con $1.8 \mathrm{mg} / \mathrm{l}$ y $1.1 \mathrm{mg} / \mathrm{l}$ en El Mollar (Fig. 4D y F). En Los Sosa el manganeso en general fue superior a $0.1 \mathrm{mg} / \mathrm{l}$ durante el período estudiado, alcanzando un máximo de $0.3 \mathrm{mg} / \mathrm{l}$ en octubre/01 (Fig. 4G). Las determinaciones de cadmio y cinc fueron constantes para todos los ríos, siendo los valores $<0.01$ y $0.05 \mathrm{mg} / \mathrm{l}$, respectivamente (Tabla 2). El coeficiente de correlación resultó altamente significativo entre cobre y manganeso con el hierro (Tabla 3). Los Sosa se diferenció por tener correlaciones entre cobre y profundidad $(r=-0.93 ; p<0.05)$ y entre manganeso y $\mathrm{pH}(r=-0.96 ; p<0.01)$.

En el ACP se observó que los tres primeros componentes explicaron el $57 \%$ de la variabilidad de los datos, siendo los tres primeros ejes responsables del 31,16 y $10 \%$ de la varianza total. El componente 1 ordenó hacia la parte negativa, a todas las muestras de los ríos Blanquito y de La Ovejería, entre otras, y hacia la parte positiva las del río Los Sosa, la mayoría de Tafí y de Las Carreras (Fig. 5A). Las variables del eje 1 que aportaron a esta ordenación fueron bicarbonato, sodio, calcio, magnesio, potasio y conductividad (intra-set correlations coefficients: $0.92,0.91,0.88,0.87,0.85,0.78$ y 0.74, respectivamente) (Fig. 5B). 
Tabla 3. Matriz de correlación entre las variables que presentaron nivel de significancia ** $p<0.01, * p<0.05$. Correlation matrix between the variables that showed significant level $* * p<0.01, * p<0.05$.

\begin{tabular}{|c|c|c|c|c|c|c|c|c|c|c|c|c|c|c|c|c|c|}
\hline & Prof. & Tem. & $\mathrm{pH}$ & Cond. & OD & $\mathrm{DBO}_{5}$ & $\mathrm{Cu}^{2+}$ & $\mathrm{Fe}^{3+}$ & $\mathrm{Mn}^{2+} \mathrm{HCO}_{3}^{-}$ & $\mathrm{Cl}^{-}$ & $\mathrm{SO}_{4}^{2-}$ & $\mathrm{Ca}^{2+}$ & $\mathrm{Mg}^{2+}$ & $\mathrm{Na}^{+}$ & $\mathrm{K}^{+}$ & $\mathrm{NO}_{2}^{-}$ & $\mathrm{NO}_{3}^{-} \mathrm{PO}_{4}^{3-}$ \\
\hline Prof. & 1 & & & & & & & & & & & & & & & & \\
\hline Tem. & $.36^{*}$ & 1 & & & & & & & & & & & & & & & \\
\hline $\mathrm{pH}$ & $.44 * *$ & $.52 * *$ & 1 & & & & & & & & & & & & & & \\
\hline Cond. & & & & 1 & & & & & & & & & & & & & \\
\hline OD & & $-.58^{* *}$ & & $.53^{* *}$ & 1 & & & & & & & & & & & & \\
\hline $\mathrm{DBO}_{5}$ & & & & & & 1 & & & & & & & & & & & \\
\hline $\mathrm{Cu}^{2+}$ & & $.38^{*}$ & & & & & 1 & & & & & & & & & & \\
\hline $\mathrm{Fe}^{3+}$ & & $.37 *$ & & & $-.47 * *$ & & $.46^{* *}$ & 1 & & & & & & & & & \\
\hline $\mathrm{Mn}^{2+}$ & & & & & & & & $.45^{* *}$ & 1 & & & & & & & & \\
\hline $\mathrm{HCO}_{3}^{-}$ & & & $.55 * *$ & $.60 * *$ & & & & & 1 & & & & & & & & \\
\hline $\mathrm{Cl}^{-}$ & $.36^{*}$ & $.52 * *$ & $.48 * *$ & $.38 *$ & & & & & $.73^{* *}$ & 1 & & & & & & & \\
\hline $\mathrm{SO}_{4}^{-}$ & & & & & & $.36^{*}$ & & & & & 1 & & & & & & \\
\hline $\mathrm{Ca}^{2+}$ & & & $.46^{* *}$ & $.79 * *$ & $.41 *$ & & & & $.83 * *$ & $.57^{* *}$ & & 1 & & & & & \\
\hline $\mathrm{Mg}^{2+}$ & $.35^{*}$ & & $.45^{* *}$ & $.67 * *$ & & & & & $.81^{* *}$ & $.70^{* *}$ & & $.73^{* *}$ & $=1$ & & & & \\
\hline $\mathrm{Na}^{+}$ & & & $.41^{*}$ & $.77 * *$ & & & & & $.85^{* *}$ & $.66^{* *}$ & & $.90^{* *}$ & $.78^{* * *}$ & 1 & & & \\
\hline $\mathrm{K}^{+}$ & & & $.51 * *$ & $.48^{* *}$ & & & & & $.84 * *$ & $.66 * *$ & & $.72 * *$ & $.62 * *$ & $.75 * *$ & 1 & & \\
\hline $\mathrm{NO}_{2}^{-}$ & & & & & & & & & & & & & & & $.46^{* *}$ & 1 & \\
\hline $\mathrm{NO}_{3}^{-}$ & $-.56^{* *}$ & & & & & & & & & & & & & & & & 1 \\
\hline $\mathrm{PO}_{4}^{3-}$ & & & & & & & & & & & $.37 *$ & & & & $.42^{*}$ & & 1 \\
\hline
\end{tabular}
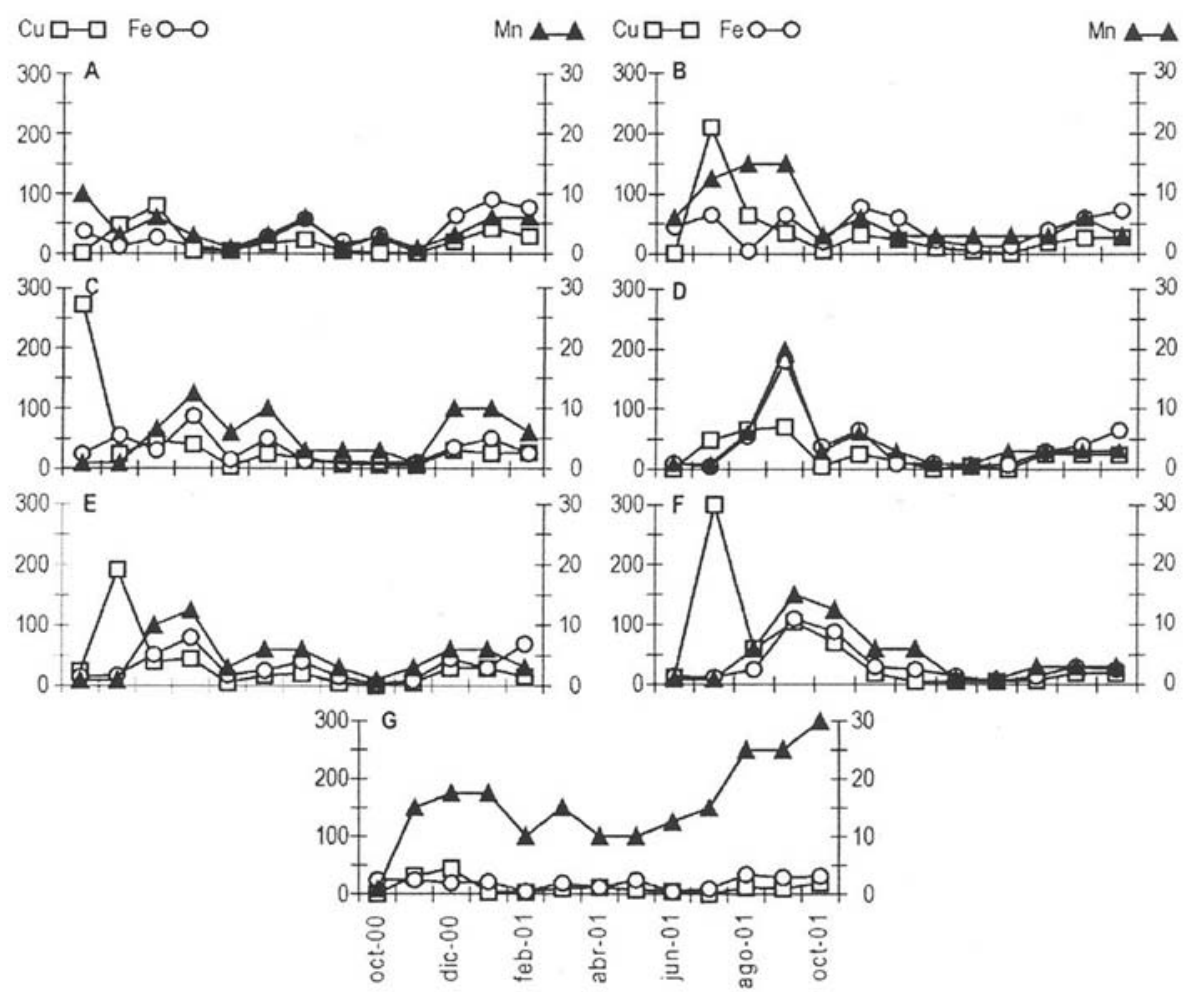

Figura 4. Variación anual de las concentraciones de cobre, hierro y manganeso $\left(\mathrm{mg} / \mathrm{l} \cdot 10^{2}\right)$ : A-R. Blanquito; B-R. Churqui; C-R. de Las Carreras; D-R. de La Ovejería; E-R. Tafí; F-R. El Mollar y G-R. Los Sosa. Annual variation of copper, iron, and manganese concentrations $\left(\mathrm{mg} / \mathrm{l} \cdot 10^{2}\right)$ : A-R. Blanquito; B-R. Churqui; C-R. de Las Carreras; D-R. de La Ovejería; E-R. Tafí; F-R. El Mollar and G-R. Los Sosa. 

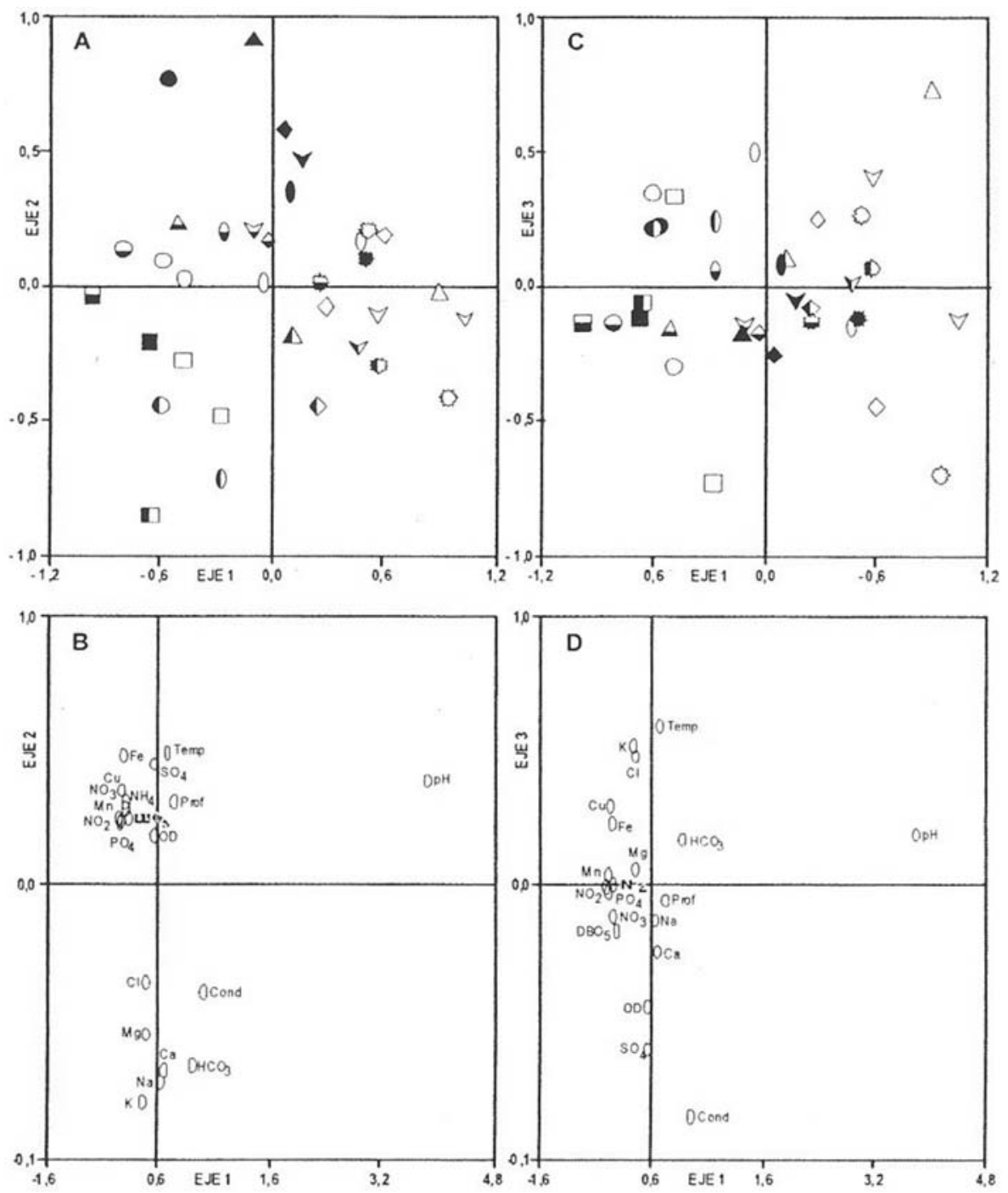

Figura 5. Análisis de componentes principales (ACP) de los ríos en relación con las variables físicas y químicas: A y B: Representación de los Componentes 1 y 2; C y D: Representación de los Ejes 1 y 3. Símbolos: períodos estudiados: verano (negro), otoño (mitad inferior negra), invierno (mitad izquierda negra) y primavera (blanco). Principal Components Analysis (PCA) of the rivers in relation to the physical and chemical variables: $A$ and B: Representation of the Components 1 and 2; $C$ and D: Representation of the axes 1 and 3. Symbols: $\square-R$. Blanquito; $O-R$. Churqui; $\diamond-R$. de Las Carreras; $\bigcirc-R$. de La Ovejería; $\vee-R$. Tafí; $\triangle$-R. El Mollar y -R. Los Sosa; studied periods: summer (black), autumn (inferior half black), winter (left half black) and spring (white).

En cuanto al componente 2, separó las muestras de verano y otoño (con excepción del río Blanquito) hacia la parte positiva y todas las de invierno y la mayor parte de las de primavera del 2000 y 2001 hacia la negativa (Fig. 5A). Los parámetros que colaboraron en la ordenación con respecto al componente 2 fueron: temperatura, $\mathrm{OD}$, hierro, cobre y manganeso (intra-set correlations coefficients: $0.85,-0.69$, $0.68,0.56$ y 0.51 , respectivamente) (Fig. 5B).

El componente 3 ordenó hacia la parte positiva a las muestras de invierno de cinco ríos, excepto Blanquito y de Las Carreras, y las de primavera del 2001 y negativamente a la mayoría de las de otoño, verano y primavera del 2000 (Fig. 5C). Las variables del 
eje 3 que aportaron a la distribución de las muestras fueron: sulfato, $\mathrm{DBO}_{5} \mathrm{y}$ amonio (intraset correlations coefficients: $0.78,0.64$ y -0.50 , respectivamente) (Fig. 5D).

\section{DISCUSIÓN Y CONCLUSIONES}

Vannote et al. (1980) consideran la ecología de los ríos como el estudio de las variaciones y procesos hidrológicos y ecológicos en el marco del "río como un continuo". En los últimos años, este concepto ha sufrido cambios al reconocer la heterogeneidad espacial y temporal de estos ambientes lóticos (Pringle et al., 1988). Esta situación se complica aún más cuando se trata de interpretar los procesos que ocurren en ríos de montañas de zonas subtropicales. En el valle de Tafí en los últimos años se incrementó la población y la actividad agrícola-ganadera, produciéndose alteraciones antrópicas por falta de programas de manejo adecuados (Locascio de Mitrovich et al., 1997, entre otros).

La época de estiaje, comprendida entre mayo y octubre de 2001, coincidió consecuentemente con los bajos valores de profundidad y velocidad de la corriente. Se debe tener en cuenta que los ríos presentan aguas someras, por lo que se observó correlación significativa entre la temperatura y la profundidad.

La temperatura influye en la solubilidad de la mayoría de las sales y sobre todo en los gases, incidiendo así en la conductividad y en el pH del agua (Moraña, 1998). En la Tabla 3 y en las correlaciones individuales de los ríos estudiados se demostraron estos efectos.

En relación a la conductividad, de acuerdo a Rodier (1990), se puede clasificar el río de Las Carreras como de mineralización muy débil, Los Sosa media acentuada y los restantes cursos de agua débil.

El escaso caudal de los ríos en época de estiaje fue incapaz de diluir el aporte iónico de las aguas residuales de las poblaciones vecinas, registrándose los valores más elevados de la conductividad, lo que coincide con lo señalado por Moraña (1998) para la cuenca hidrográfica del río Mojotoro, Salta. Las mediciones obtenidas de este parámetro (Fig. 2A-G) concuerdan con el río Gastona, Tucumán (Mirande et al., 1999), la Cuenca alta del río Bermejo, Jujuy (Moraña et al., 2000, entre otros), mientras que presentaron registros mayores una gran parte de los ríos del noroeste argentino (Fernández et al., 2002; Moraña, 1998; Salusso, 1998, 2000 y Villagra de Gamundi et al., 1993).

El oxígeno disuelto está en función del origen del agua. En las aguas superficiales pueden presentar valores próximos a la saturación, conteniendo $9.1 \mathrm{mg} / \mathrm{l}$ de oxígeno a $20{ }^{\circ} \mathrm{C}$ y a presión normal (Rodier, 1990). Las concentraciones del oxígeno disuelto en los ambientes lóticos estudiados fluctuaron entre los límites permitidos por las normas vigentes de calidad de agua para potabilización, irrigación y esparcimiento.

Los registros de la demanda bioquímica de oxígeno, al no superar los $2 \mathrm{mg} / \mathrm{l}$, se asemejan a los encontrados en zonas prístinas (Mirande et al., 1999; Moraña, 1998 y Salusso, 1998).

La concentración de los iones mayoritarios muestra una correlación mutua elevada y tanto su conjunto como los valores de cada uno de ellos están positivamente correlacionados con la conductividad del agua (Tabla 3). Estos elementos se pueden considerar como componentes principales de proporcionalidad relativamente constante, que dan al agua su carácter y cuyas proporciones son poco influidas por la actividad de los organismos (Margalef, 1983). De acuerdo a la concentración iónica se puede caracterizar al agua de todos los ríos analizados como bicarbonatadascálcicas concordando con la composición relativa de las aguas dulces así como de los ríos de todo el mundo (Margalef, 1983). Asimismo, estas características del agua coinciden con la litología de esta zona montañosa, ya que las precipitaciones no disuelven fácilmente las sales de las rocas del basamento metamórfico ni del granito (Tineo et $a l ., 1998)$ y en consecuencia de ésto, la conductividad eléctrica es relativamente baja.

Los compuestos nitrogenados y ortofosfatos se presentaron en bajas concentraciones, lo que se debe de acuerdo a Pesson (1979), a la ausencia de aportes exógenos que afecten las aguas naturales.

Los metales pesados arsénico, cobre, hierro y manganeso registraron valores, a veces elevados, 
aunque se consideran normales para cursos de aguas naturales (Rodier, 1990). Los máximos registros de hierro que se presentaron en el río de La Ovejería y de cobre en el río El Mollar, se deberían a la contaminación antropogénica por el uso de pesticidas y fertilizantes que contienen entre sus componentes, dichos cationes.

La interpretación de los análisis de ordenación realizados sobre la matriz de parámetros físicos y químicos, permitió diferenciar a los sistemas lóticos temporal y espacialmente. El componente 1 ordenó a los puntos de muestreo de acuerdo a los iones mayoritarios predominantes y a la conductividad, lo que podría interpretarse como un eje de mineralización. Si consideramos los componentes 2 y 3 , separaron las muestras de acuerdo a la variación cíclica estacional. Ambos ejes ordenaron las de invierno y primavera y las de verano y otoño, lo que coincidió con las condiciones climáticas del área de estudio.

\section{AGRADECIMIENTOS}

Agradecemos al Consejo de Investigaciones de la Universidad Nacional de Tucumán (CIUNT, G335/1) y al Consejo Nacional de Investigaciones Científicas y Técnicas (CONICET, PIP 0871/98) por los subsidios otorgados para realizar esta investigación.

\section{BIBLIOGRAFÍA}

ACOSTA M., M. J. AMOROSO, M. A. BARRIONUEVO, C. BUTÍ, F. CANCINO, A. ECHAVARRÍA, J. GIMENEZ DE PÁEZ, J. JUÁREZ, C. LOCASCIO DE MITROVICH, M. L. MATTASSINI, G. S. B. MEONI, M. G. NAVARRO, N. ROMERO, C. SEELIGMANN, M. SIROMBRA, A. B. A SOSA, B. C. TRACANNA, F. H. VENDRAMINI y A. VILLAGRA DE GAMUNDI. 2002. Aspectos preliminares sobre la biota y la contaminación en el embalse La Angostura (Tucumán, Argentina). Actas I Congreso Iberoamericano de ambiente y calidad de vida. $2^{\circ}$. Congreso de ambiente y calidad de vida, Catamarca, Argentina: 245.

ALDERETE, M. C. 1998. Unidades fisiográficas. En: Geología del Tucumán. M. Gianfrancisco, M. E.
Puchulu, J. Durango de Cabrera y G. F. Aceñolaza (eds.): 29-40. $2^{\mathrm{a}}$ ed. Colegio de Graduados de Ciencias Geológicas, Tucumán.

APHA. 1992. Standard methods for the examination of water and wastewater. $18^{\text {th }}$ ed. American Public Health Association, Washington. $710 \mathrm{pp}$.

BUCHER, E. H. y J. M. CHANI. 1998. Chaco. En: Los Humedales de la Argentina: Clasificación, Situación actual, Conservación y Legislación. Canevari, P., D. E. Blanco, E. H. Bucher, G. Castro \& I. Davidson (eds.): 75-96. Wetlands Internacional Publ. 46, Buenos Aires, Argentina.

CABRERA, A. 1976. Regiones Fitogeográficas Argentinas. Enciclopedia Argentina de Agricultura y Jardinería, 2(1): 1-85. Ed. ACME, 2da. ed., Buenos Aires, Argentina.

CANEVARI, P., D. E. BLANCO, E. BUCHER, G. CASTRO y I. DAVIDSON. 1998. Los Humedales de la Argentina. En: Los Humedales de la Argentina: Clasificación, Situación actual, Conservación y Legislación. Canevari, P., D. E. Blanco, E. H. Bucher, G. Castro \& I. Davidson (eds.): 324. Wetlands Internacional Publ. 46, Buenos Aires, Argentina.

FERNÁNDEZ, H., F. ROMERO, M. B. VECE, V. MANZO, C. NIETO y M. ORCE. 2002. Evaluación de tres índices bióticos en un río subtropical de montaña (Tucumán-Argentina). Limnetica, 21(2): 1-13.

GARCIA SALEMI, M. A. 1974. El valle de Tafi, evolución geomorfológica y planeamiento hidrológico. Tesis Doctoral, Universidad Nacional de Tucumán. 64 pp.

LOCASCIO DE MITROVICH, C., B. TRACANNA, A. VILLAGRA DE GAMUNDI, C. SEELIGMANN y C. BUTÍ. 1997. Situación actual de la problemática limnológica de los embalses de la provincia de Tucumán (Argentina). Lilloa, 39(12): 81- 93.

MARGALEF, R. 1983. Limnología. Barcelona: Omega. $1010 \mathrm{pp}$.

MIRANDE, V., N. ROMERO, M. A. BARRIONUEVO, G. S. MEONI, M. G. NAVARRO, M. C. APELLA \& B. C. TRACANNA. 1999. Human impact on some limnological characteristics of the Gastona river (Tucumán, Argentina). Acta Limnologica Brasiliensia, 11(2): 101-110.

MORAÑA, L. B. 1998. Estudio de la calidad del agua en un subsistema de ríos de la provincia de Salta sometidos a acción antrópica. Tesis 
de Magister, Universidad Nacional del Litoral. Argentina. $88 \mathrm{pp}$.

MORAÑA, L. B., M. M. SALUSSO y M. O. GARCIA DE EMILIANI. 2000. Evaluación de la calidad del agua en la cuenca del río Grande (Alta Cuenca del Bermejo, Jujuy) Argentina. En: Uso y preservación de los recursos hídricos en los umbrales del siglo XXI. H. D. Farías, M. T. Pilán, M. J. Borsellino, F. J. Pece, A. R. Storniolo y M. T. Mattar (eds.): 285-286. Instituto de Recursos Hídricos, Facultad de Ciencias Exactas y Tecnologías, Universidad Nacional de Santiago del Estero, Argentina.

PERONDI, M. E., M. C. GALINDO, M. B. VECE, M. C. APELLA y M. del V. HIDALGO. 1996. Relevamiento de variables físicoquímicas del río Medina- Provincia de Tucumán. Serie Monográfica y Didáctica 31, Fac. Ciencias Naturales e Instituto Miguel Lillo, Universidad Nacional de Tucumán, Argentina. 25 pp.

PESSON, P. 1979. La contaminación de las aguas continentales. Incidencias sobre las biocenosis acuáticas. Madrid. Mundi-Prensa. 335 pp.

PRINGLE, C. M., R. J. NAIMAN, G. BRETSCHKO, J. R. KARR, M. W. OSWOOD, R. J. WEBSTER, R. I. WELCOMM \& M. J. WINTERBOURN. 1988. Match dynamics in lotic systems: The stream as a mosaic. J. N. Am. Benthol. Soc., 7: 503-524.

RODIER, J. 1990. Análisis de las aguas. Barcelona: Omega. 1059 pp.

ROHLF, F. J. 1990. NTSYS-pc. Numerical taxonomy and multivariate analysis system ver. 1.60. Exeter Software, New York. 159 pp.

SALUSSO, M. M. 1998. Evaluación de la calidad del agua de dos ríos del Valle de Lerma (Salta) sometidos a acción antrópica. Tesis de Magister, Universidad Nacional del Litoral. Argentina. $70 \mathrm{pp}$.

SALUSSO, M. M. 2000. Variabilidad temporo-espacial en la calidad del agua del río Juramento (Cuenca Superior, Salta). En: Uso y preservación de los recursos hídricos en los umbrales del siglo XXI. H. D. Farías, M.T. Pilán, M.J. Borsellino, F.J. Pece, A.R. Storniolo y M.T. Mattar (eds.): 299-300. Intituto de Recursos Hídricos, Facultad de Ciencias Exactas y Tecnologías, Universidad Nacional de Santiago del Estero, Argentina.

SAYAGO, J. M., M. M. COLlANTES y M. A. TOLEDO. 1998. Geomorfología. En: Geología del Tucumán. M. Gianfrancisco, M. E. Puchulu, J.
Durango de Cabrera y G.F. Aceñolaza (eds.): 241257. 2da. ed. Colegio de Graduados de Ciencias Geológicas, Tucumán.

SEELIGMANN, C. T. 1999. Dinámica del fitoplancton del río Salí (Tucumán, Argentina) en relación a la contaminación. Natura Neotropicalis, 30(1-2): 57-66.

SEELIGMANN, C. T., B. C. TRACANNA y S. ISASMENDI. 2003. Fitoplancton de un embalse de alta cota (Tucumán, Argentina). Bol. Soc. Argent. Bot., 38 (Suplemento): 165.

SEELIGMANN, C., B. C. TRACANNA, S. MARTINEZ DE MARCO y S. ISASMENDI. 2001. Algas fitoplanctónicas en la evaluación de la calidad del agua de sistemas lóticos en el noroeste argentino. Limnetica, 20(1): 123-133.

SEELIGMANN, C., A. VILLAGRA DE GAMUNDI, B. C. TRACANNA, C. LOCASCIO DE MITROVICH y J. JUAREZ. 2004. Comunidad planctónica en un embalse de alta cota (TucumánArgentina). Resúmenes II Reunión Binacional de Ecología, XXI Reunión Argentina de Ecología y XI Reunión de la Sociedad de Ecología de Chile, Mendoza, Argentina: 183.

TINEO, A., C. M. FALCÓN, J. W. GARCÍA, C. H. D'URSO, G. GALINDO y G. V. RODRIGUEZ. 1998. Hidrogeología. En: Geología del Tucumán. M. Gianfrancisco, M. E. Puchulu, J. Durango de Cabrera y G. F. Aceñolaza (eds.): 259-274. 2da. ed. Colegio de Graduados de Ciencias Geológicas, Tucumán, Argentina.

TRACANNA, B.C. y S. N. MARTINEZ DE MARCO. 1997. Ficoflora del río Salí y sus tributarios en áreas del Embalse Dr. C. Gelsi (TucumánArgentina). Natura Neotropicalis, 28(1): 23-38.

TRACANNA, B. C. y C. SEELIGMANN. 2004. Variaciones espaciales y temporales del fitoplancton del embalse La Angostura (Tucumán-Argentina). Resúmenes I Congreso Latinoamericano sobre Biotecnología Algal, Buenos Aires, Argentina: 108.

TRACANNA, B. C., C. T. SEELIGMANN, V. MIRANDE, L. B. DE PARRA, M. T. DE PLAZA y F. MOLINARI. 1999. Cambios espaciales y temporales del fitoplancton en el Embalse Río Hondo (Argentina). Bol. Soc Argent. Bot., 34 (1-2): 101108.

VANNOTE, R. L., G. W. MINSHALL, K. W. CUMMINS, J. R. SEDELL \& C. E. CUSHING. 1980. The river continuum concept. Can. J. Fish. Aquat. Sci., 37: 130-137. 
VERVOORST, F. 1981. Mapa de las Comunidades vegetales de la provincia de Tucumán. En: Lista de los anfibios y reptiles de la provincia de Tucumán. R. Laurents y E. Terán (eds.): 8-9, Miscelánea 71. Fundación Miguel Lillo. Tucumán, Argentina.

VILLAGRA DE GAMUNDI, A., C. SEELIGMANN
DE SOSA GOMEZ, B. C. TRACANNA y C. LOCASCIO DE MITROVICH. 1993. Sobre la limnología físicoquímica del río Salí y sus tributarios en áreas próximas al Embalse El Cadillal (Tucumán-Argentina). Miscelánea 91. Fundación Miguel Lillo. Tucumán, Argentina. 18 pp. 
